\title{
Design and operational control of an AGV system
}

\author{
Ronald J. Mantel*, Henri R.A. Landeweerd \\ Production and Operations Management Group, Department of Mechanical Engineering, University of Twente, P.O. Box 217, \\ 7500 AE Enschede, The Netherlands
}

Accepted for publication 11 January 1995

\begin{abstract}
In this paper we first deal with the design and operational control of Automated Guided Vehicle (AGV) systems, starting from the literature on these topics. Three main issues emerge: track layout, the number of AGVs required and operational transportation control. An hierarchical queueing network approach to determine the number of AGVs is decribed. Also basic concepts are presented for the transportation control of both a job-shop and a flow-shop. Next we report on the results of a case study, in which track layout and transportation control are the main issues. Finally we suggest some topics for further research.
\end{abstract}

Keywords: AGV system; Track layout; Number of vehicles; Transportation control

\section{Introduction}

Automated Guided Vehicles (AGVs) are unmanned means of inplant transportation, which are used in both production and assembly areas and warehouses. Two types of AGVs should be distinguished: free-ranging and path-restricted vehicles. The first type uses dead reckoning and laser or infrared light equipment, where the light is reflected by 'mirrors' on the walls to determine its position. Also a grid pattern on the floor may be used for callibration. This is created by transponders or magnets or by a chess board pattern that is optically scanned. The second type of AGV is restricted

* Corresponding author. to a fixed network path structure (track layout), guided by either induction wires in the floor, or by a paint or tape track on the floor, that is optically followed.

The technical control is performed by PLCs and mostly the power is supplied by a battery. The traffic control to prevent collisions is either realized through splitting the tracks into zones, in each of which only one AGV is allowed at a time, or by sensors.

Sections 2 and 3 deal with AGV system design and operational control, starting from the literature on these topics, where also is tried to structure the main issues involved. In Section 3 we also present basic concepts for inplant transportation control. Next, the results of a case study are reported in Section 4. Finally, in Section 5, topics for further research are mentioned. 


\section{AGV system design}

\subsection{Design issues}

It may be noticed that many of the issues of an AGV system are also applicable on a fleet of fork lift trucks, especially if they are supplied with Radio Frequency (RF)-communication equipment.

King and Wilson [1], Tanchoco and Co [2] and Srinivasan et al. [3] already give in their papers extensive reviews of the literature on AGV system design and control. In this section it is tried to structure the issues involved and their mutual relations.

From the literature three design issues emerge: track layout, the number of AGVs required and operational transportation control. These topics together determine the transportation capacity (maximum throughput per unit of time) of an AGV system. Next they will be discussed one by one.

\subsubsection{Track layout}

It is obvious that for path-restricted AGVs a well-designed track layout (a good infrastructure, i.e. a network path structure with short distances, efficiently used paths with little blocking (congestion) and well-dimensioned in- and output buffers with minimal waiting times at the stations, is very important. Although the routing of a free-ranging $\mathrm{AGV}$ is more flexible, also for that type of vehicle in most cases a track layout will be defined in order to limit the number of possible routes, facilitating the operational control.

In most papers on AGV systems the track layout is assumed to be given and only the number of AGVs required and operational control are subjects of study. However, there are a number of papers, in which the track layout as well as uni- or bidirectional use of paths, the locations of pick-up and drop-off stations and the sizes of input and output buffers are addressed [4-7]. A recent trend is the so-called tandem configuration [8], i.e. a track layout consisting of independent simple loops with only one AGV assigned to each loop and load transfer stations between the loops (see Fig. 1). Also single loops have been studied [9]. Such configurations have the advantage that no congestion occurs and their control is rather simple. A disadvantage is that probably more vehicles are needed than in a conventional configuration.

\subsubsection{The number of $A G V S$ required}

The number of AGVs required is the sum of the total loaded and empty travel time and waiting time (among other things due to congestion) of the AGVs in a busy time period, divided by the time an $\mathrm{AGV}$ is available during that period. As this concerns a design issue, the calculation may be rough; e.g. in [6] no waiting time is taken into account. In that paper the empty travel time is minimized by means of an LP model. Next we will present a

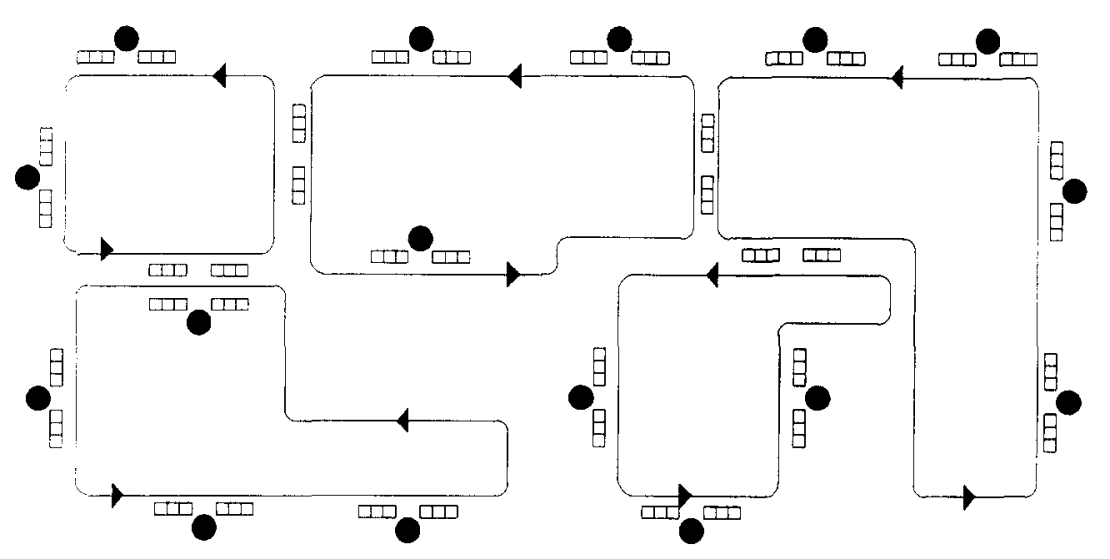

Fig. 1. Tandem configuration (reprint from [1]). 
hierarchical queueing network model to determine quickly and roughly the number of AGVs required. For other approaches we refer to [3].

Hierarchical queueing network tool. For instance, it is interesting to make a quick comparison between a tandem configuration and a conventional track layout as to the number of AGVs required. For this purpose we developed a queueing network analysis tool for a rapid determination of this number, given a track layout. What is new in this approach is that it is an hierarchical one, that consists of two levels. No iterations between these levels are needed. A weak point is that the empty travel, though crucial, cannot be taken into account yet.

At the upper level the transportation tasks are offered to a set of AGVs that are modelled as a set of parallel servers. Here transportation time is the process time. At the lower level AGV trips are offered to zones that are modelled as single servers with a deterministic process (i.e. transportation) time. Because of the restriction that we only deal with single-load AGVs (see Section 2.1.3), we assume that the arrival processes at both levels are equivalent if there are sufficient AGVs available.

Start with one AGV. Then the procedure is as follows. First the queueing network problem at the lower level is solved. Next the result, the travel times between the stations, is used at the upper level. If the number of AGVs available is too small to handle the job intensity, then increase this number by 1 and carry out the same procedure. Stop if the number of AGVs is sufficient.

For a tandem configuration a more simple, onelevel, approach can be adopted.

\subsubsection{Operational transportation control}

Operational transportation control consists of two hierarchical levels: job control and traffic control. The first mentioned, upper, level routes and schedules the AGVs, given the transportation tasks to be performed. The second, lower, level consists of simple traffic rules (e.g. an AGV that comes from the right goes first). In Section 3.2 we will come back to the interaction between those two levels.
In Section 3 first job control will be treated. This control generally aims at the minimisation of total empty travel time and waiting time, thus reducing transportation lead times. Also buffer occupancies may be taken into account here. The literature on AGV control almost exclusively deals with the control of single-load vehicles, i.e. vehicles that can carry only one unit load, e.g. a pallet or a tote, at a time. To our knowledge the control of multipleload vehicles is only addressed in a few papers [10-12]. It is obvious that the transportation control of multiple-load vehicles is much more complex. Probably, models and methods from vehicle routing in physical distribution can be used, e.g. the so-called dial-a-ride problem and the pick-up and delivery problem. It should be noted that in this paper only single-load vehicles will be considered. Due to this restriction the pattern of an AGV route is such that a loaded trip is always followed by an empty trip, vice versa.

It exists various terms to classify AGV operational control policies. An overview is given in Fig. 2. These terms will be explained in Section 3.

\subsection{Relations between the issues}

The relations between the main issues, mentioned in the preceding sections are shown in Fig. 3. From this it is clear that both loaded travel time and empty travel time depend on the track layout.

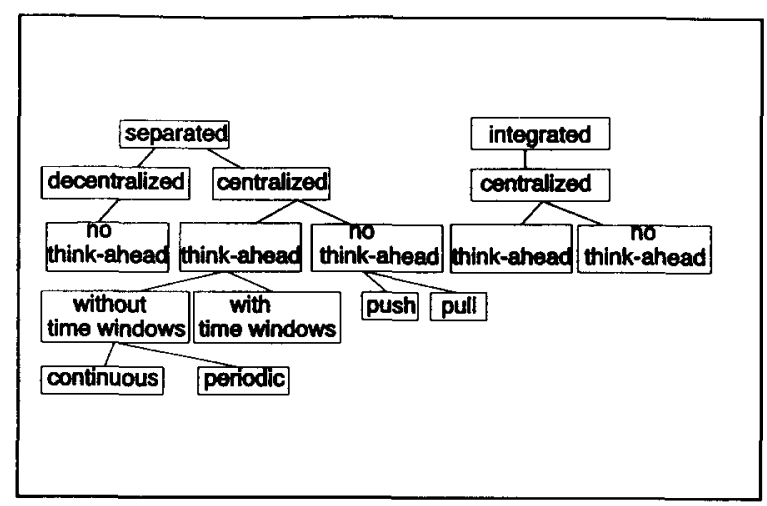

Fig. 2. Taxonomy of terms. 


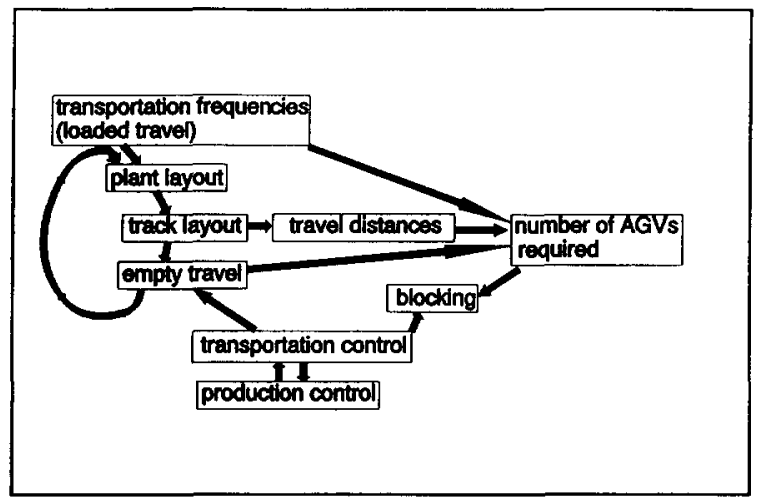

Fig. 3. Relations between the main issues of AGV system design.

\subsection{Performance indicators}

Performance indicators which may be used to compare alternative layouts and operational control policies are, e.g. the throughput, transportation lead times, the makespan of an order set and robustness against dead-lock situations. A diagram, indicating the work load as a function of time and the lead time for each product in each subsystem (e.g. the transportation system, the buffers and the machines), gives insight in the dynamic behaviour of a system and so this can be a useful tool for performance analyses.

\section{Operational control}

\subsection{Job control}

\subsubsection{Interaction between production and transportation control}

First, it is important to decide upon the interaction between the operational control of the transportation system and the operational control of the production system. The structure of the production system determines to a great extent the selection of the production and transportation control strategy. One should distinguish two main structures: the flow-shop and the job-shop configuration.

It may be decided to control production and transportation separately (e.g. schedule production tasks first and, taking the resulting time scheme as a starting point, subsequently schedule the transportation tasks required) or to integrate both control activities. The interaction between production and transportation control seems to attract little attention in the literature. Mostly they are separated; in fact most authors assume a certain arrival pattern for transportation tasks and restrict themselves to the control of the transportation system.

\subsubsection{Centralized/decentralized control}

The transportation control is either centralized or decentralized. A centralized control implies that all transportation tasks are concurrently considered when the vehicles to perform them are routed and scheduled. In contrast, the First Encountered First Served (FEFS) rule (i.e. a vehicle makes a tour and performs the first transportation task it encounters, in other words an AGV looks for work) is a decentralized way of control, that is typically suited for a single-loop and a tandem configuration. In the remainder of this section we will restrict ourselves to centralized control policies.

\subsubsection{Think-ahead/no think-ahead control}

Another classification of transportation control is to distinguish think-ahead and no think-ahead policies. Think-ahead implies that known tasks are combined to routes such that empty travel time is minimized.

\subsubsection{Policies for transportation control}

Combining two of the characteristics, mentioned above, leads to four main different policies for transportation control, that will be discussed successively:

(i) separated/no think-ahead control,

(ii) integrated/no think-ahead control,

(iii) separated/think-ahead control,

(iv) integrated/think-ahead control.

(i) In this case often a centralized control, that consists of a couple of simple 'no think-ahead' rules, one for transportation task assignment and the other for idle vehicle assignment, is adopted. The system is on-line monitored. Two possible situations arise. First there is the situation that a task arrives while all vehicles are busy. Then it is added to a list of 
tasks, waiting for an AGV that becomes idle. Task assignment means that one of the tasks on this list, according to a certain priority, is assigned to a vehicle that becomes idle. Another possible situation is that a set of vehicles is idle, waiting for a task. Idle vehicle assignment means that one of the vehicles from this set, according to a certain priority, is assigned to a task that arrives. Note that only one of both situations can occur at a time. It may be clear that these rules are also called dispatching rules (dispatching implies that a vehicle is ordered to perform a task).

A transportation task assignment rule can be either a push or a pull type rule, where respectively the output buffer or the input buffer of a station controls the priority of a transportation task. In [13] various push rules, especially suited for jobshop situations, e.g. the MROQS (minimum remaining space in an output buffer) rule, are presented and compared by simulation. Some of these rules lead to shop-locking, the phenomenon of the propagation of local deadlocks that leads to a complete standstill of the transportation system. On the other hand, $[14,15]$ deal with pull rules. The latter rules are suited for a JIT controlled flow-shop. In fact, a pull rule implies an integrated control of production and transportation.

In [13] also rules for idle vehicle assignment (e.g. Nearest Vehicle first, Longest Idle Vehicle first) are given. The latter rule balances the wear over all vehicles.

(ii) As stated before, production and transportation control are often separated, however [16] treats 'no think-ahead' rules for integrated control.

(iii) This implies a centralized policy, suited for the transportation control within job-shops, that by thinking-ahead results in an efficient use of vehicles. This policy will be treated in more detail, also because this way of control gets only little attention in the literature on AGV systems sofar $[17,18]$. Probably, as already noticed by King and Wilson [1], techniques from the domain of vehicle routing and scheduling may be used here to generate efficient routing schemes and time schedules $[19,20]$. However, the arrival rate of transportation tasks for an inplant transportation system is much higher than it is for a physical distribution system, so that for AGV system control on-line monitoring of the system and a relatively short time horizon should be adopted.

Two situations can be distinguished:

(a) Think-ahead control without time windows. The loaded trips to be carried out are known. As time windows do not play a role here, only the routing of the AGVs needs to be determined, such that total empty travel distance is minimized. If waiting time is neglected, then the problem may be formulated as a Multiple-Travelling-Salesman Problem with an asymmetric travel distance matrix, where the nodes represent the loaded trips. This results in Hamiltonian chains, AGV routes, that may vary in length considerably. If one likes to balance these lengths then a Clarke and Wright [21] type of heuristic should be adopted.

One possible option is that - at each time a new transportation task arrives - for all transportation tasks known a routing plan for the AGVs is generated (in other words tasks are assigned to AGVs). The resulting routing scheme is maintained and executed (tasks assigned are dispatched) until a new task arrives. Consequently, the arrival rate of tasks determines the nervousness of this way of control. It is possible that certain tasks stay at the 'tails' of the routes for a long time. Therefore, such tasks should be identified and get a higher priority.

Also another approach may be applied, i.e. a periodic control. This implies that during a certain time period all transportation tasks that arrive are collected and put on a list and at the end of that interval an efficient routing scheme for those tasks is generated, that has to be completely executed during the next period. This means that transportation tasks are delayed until the next period.

(b) Think-ahead control with time windows. Here it is assumed that at each moment on-line the production time schedule for a certain rolling time horizon is known, i.e. the production tasks have already been scheduled, while roughly transportation times were taken into account [22]. So time plays a crucial role here; not only routing is involved, but also scheduling. Given such a production schedule and the loaded trips (transportation tasks) due to the product routing, all feasible empty trips can be determined. The transportation lead time for both a loaded and an empty trip consists of transportation and waiting time. Next a routing 
plan and a time schedule for the available AGVs are generated, such that total empty travel time is minimized.

The example in Fig. 4 illustrates this policy. The first schedule shows all empty vehicle movements

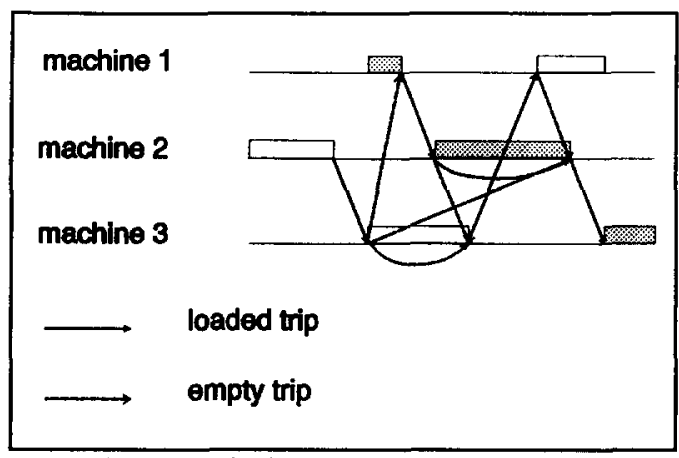

a. Production schedule and empty vehicle trips allowed

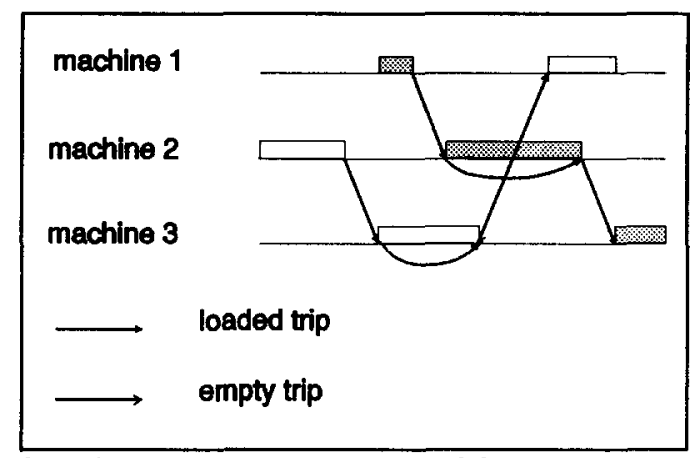

b. A feasible transportation schedule

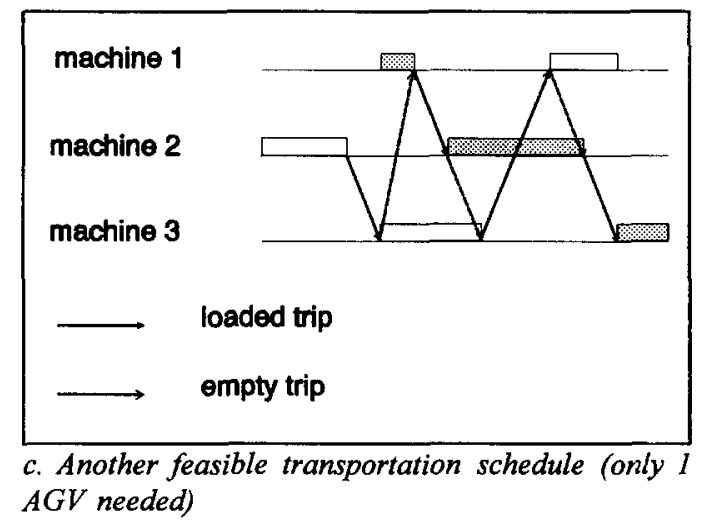

Fig. 4. AGV routing scheme and time schedule. that are feasible, given a certain production time schedule and product routing, for two products on three machines. Also in the figure two feasible transportation schedules are given. First for the case that two AGVs are available. Schedule (c) can be realized with only one AGV. Note that, as stated before, the pattern of a single-load AGV route is as follows: loaded trip, empty trip, loaded trip, empty trip, etcetera.

If there are no buffers at the stations then the routing and scheduling problem involved may be formulated as a Multiple-Travelling-Salesman Problem. If there are buffers (with infinite capacity) then the routing and scheduling problem may be modelled as a Multiple-Travelling-Salesman-Problem with time windows. Simulated annealing seems to be an appropriate solution technique for such complex combinatorial problems. If there is no feasible solution, then the production schedule must be adapted. How to handle that is still an open question.

The resulting transportation schedule is maintained and executed until a production schedule demands to make a revised one. Not only the arrival rate of transportation tasks but also the length of the time horizon determine the nervousness of this way of control.

Note: If a horizon is used, then a difficult question must be answered: how long should it be?

(iv) A possible approach for integrated control of single-load vehicles is the following. By modelling an AGV system as a set of parallel machines, with processing times equal to transportation times and by modelling empty travel times as changeover times, it could be possible to use a shop floor scheduling technique for the integrated scheduling of production and transportation. For instance the very promising, so-called Shifting Bottleneck Method [23], extended to handle parallel machines [24], may be used.

\subsection{Traffic control}

Sofar operational AGV control policies are discussed, which constitute the upper hierarchical level of operational control. As stated before, this level controls the assignment of transportation 
tasks to vehicles. Knowing that assignment, it is possible to deduce the routing and time schedule for each AGV. Then, given the track layout, one may predict where the AGVs interfere with each other, which results in congestion. The lower level of control, the traffic control, effects the amount of congestion. If this is too high, it may be tried to find blocking-free routes by rerouting the AGVs (see Fig. 5). Generally, this will lead to a revised task assignment. One of the advantages of creating blocking-free routes is that the travel time variances are reduced, so that the routing optimization problem gets a more deterministic character.

\subsection{A basic concept for operational transportation control}

Now it is tried to formulate a kind of basic concept, which may be used to control inplant transportation. Most probably, an integrated control of production and transportation with 'thinkahead' will yield the best system performance. Probably the basic concept, a much more simple approach to be discussed next, in which production and transportation are coordinated to a certain extent, will also produce good results.

As transportation can be seen as a 'slave' to production, we propose a push type of transportation control. First we discuss the concept for a jobshop situation, as illustrated in Fig. 6. When

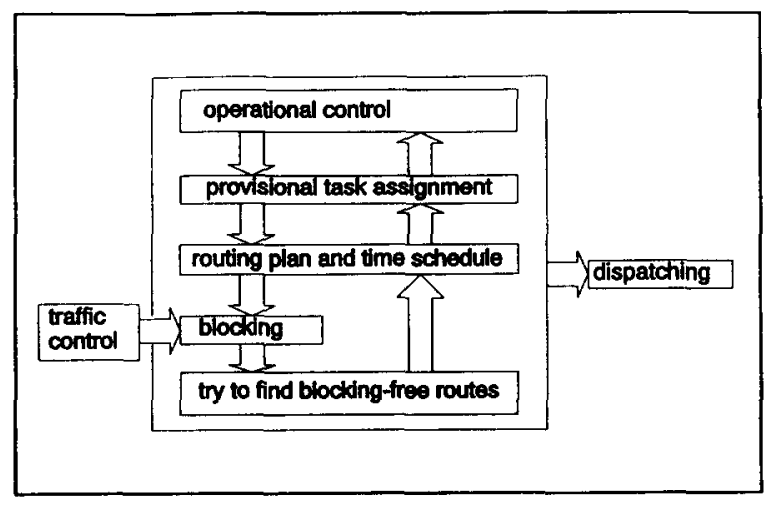

Fig. 5. Interaction between task assignment and the search for blocking-free routes.

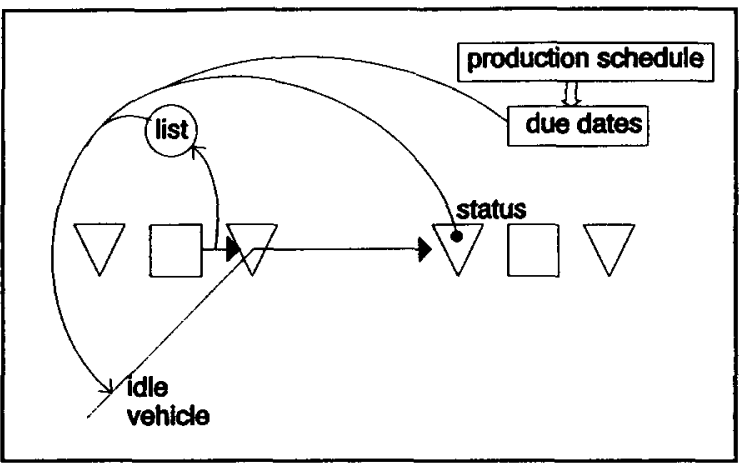

Fig. 6. Basic transportation control concept for a job-shop situation.

a product enters an output buffer of a station, centrally it is added to a list of transportation tasks to be performed. As soon as an idle vehicle becomes available, then the first transportation task on the list is assigned to that vehicle, provided that there is enough space in the input buffer of the destination and if, looking at the production schedule, the product involved is shortly to be processed at the next station. If these conditions are not met, then the next task on the list is considered. Furthermore, whenever possible, a pick-up and drop-off at a station are combined (compare this with a so-called 'dual command' in a warehouse system). Combining those actions was already mentioned by Srinivasan et al. [3].

In a flow-shop production and transportation are usually controlled in an integrated way by a pull type of policy, e.g. using kanbans. This implies that an input buffer asks the transportation system to carry Work In Process (WIP) from the preceding production stage. Only if the transportation lead times are deterministic, e.g. in case of transportation by a conveyor system, this concept will work well. If transportation takes place by means of AGVs, then the lead times are harder to predict, so that a pull policy implies that items will often arrive too late. Therefore for a flow-shop we propose to adopt an equivalent - so push - concept as just suggested for a job-shop, except that here successive production stages are linked to each other by means of kanbans (see Fig. 7). As soon as a machine asks for input to process, there goes 


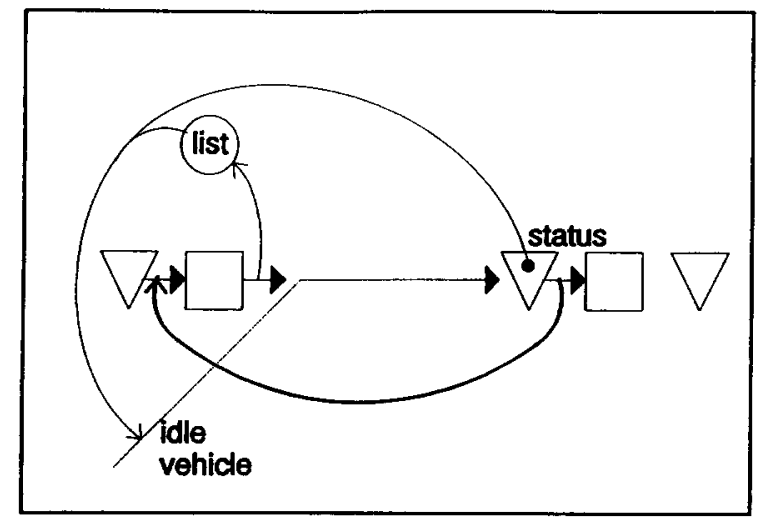

Fig. 7. Basic transportation (and production) control concept for a flow-shop situation.

a kanban to the machine of the preceding processing stage, also to ask for input.

\section{A case study}

The study reported here gives an example of separated control of production and transportation without think-ahead. Transportation control and track layout design are the main subjects of study. The problem refers to an existing AGV system in a steel company that transports coils in a job-shop environment. Traffic control is realized through zones between so-called information points. In each zone only one AGV is allowed at a time. The routing of a product is quite simple: each product is processed on only one of the machines and next it leaves the system. Therefore the current AGV system control consists of two task assignment rules: a pull rule for incoming and a push rule for outgoing coils at a station. For idle vehicle assignment the nearest vehicle rule is adopted.

One of the goals of the study was to improve the lead time performance of the system by applying alternative dispatching rules. The other aim was to determine the effect of changing the track layout (see Fig. 8(a)) slightly by splitting the network into two parts (see Fig. 8(b)), where each AGV can be assigned to only one of these parts and the AGV dispatching for each part is controlled independently.
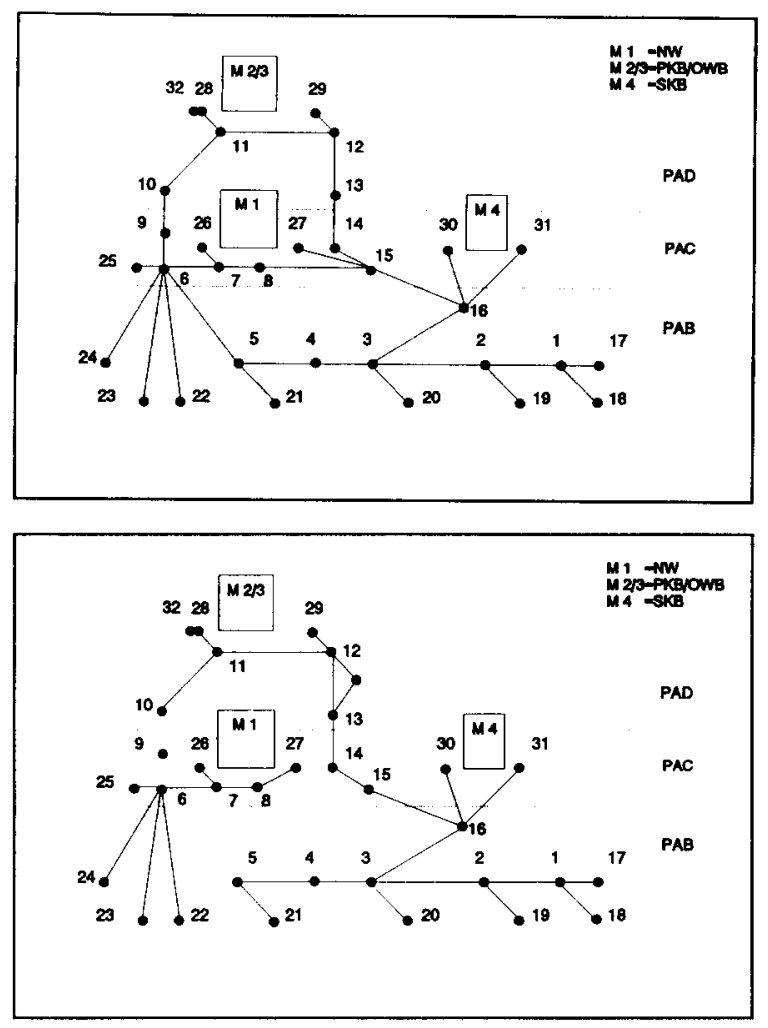

Fig. 8. (a) Current layout. Note: the dots represent information points (b) Split layout.

Several dispatching rules have been simulated, resulting in the following conclusions (see Fig. 9). Purely push dispatching rules lead to shop locking. If for the current track layout the current rules are adapted by a more efficient task assignment, i.e. to combine a drop-off and a pick-up at a station whenever possible, then the lead times decrease for most of the product types because of the reduction in total empty travel time. The performance of this policy is even better than for the case that purely pull rules are adopted.

The splitting of the track layout into two subsystems leads to the saving of one AGV, while the lead time performance even improves compared with the results for the current layout. This split layout may be seen as a step towards a tandem configuration.

Also the effects of other locations for the parking area have been investigated. Furthermore the work 


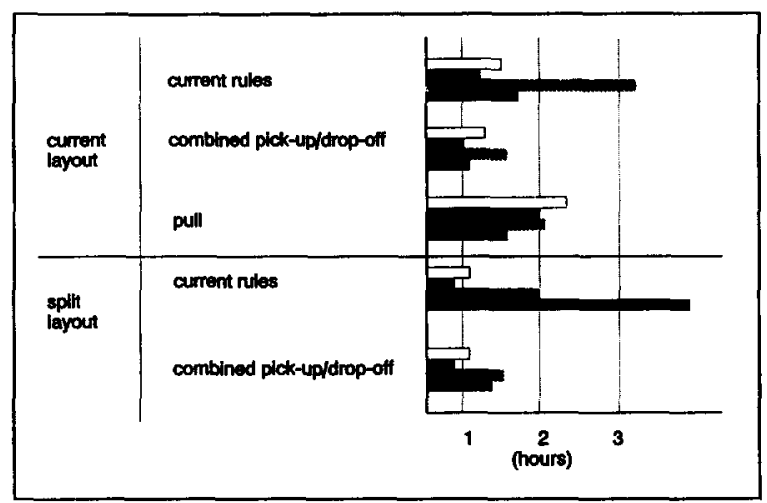

Fig. 9. Lead time performance for different track layouts and dispatching rules. (Note: a bar represents the lead time for a product type.).

load was increased by reducing the interarrival times of jobs. At a certain load level one faces the bottleneck of the entire system, i.e. then the load is equal to the capacity of the system. In the current layout one of the machines appears to be the bottleneck, where in the split layout it is the AGV system. Consequently, if travel speeds are increased (which results in shorter transportation lead times), in the split layout it is possible to further increase the work load, where in the current layout this is not possible.

\section{Topics for further research}

Topics for further research are:

- the possible benefits of free-ranging over a fixed path structure,

- a performance comparison of separated and integrated control of production and transportation,

- a performance comparison between control policies with think-ahead and policies without think-ahead,

- a comparison of a tandem configuration and a conventional track layout with regard to the number of vehicles required,

- the operational control of multiple-load vehicles,

- the possible benefits of blocking-free routing,

- applicability on AGV systems of approaches for vehicle routing and scheduling in physical distribution,
- applicability on AGV systems of control strategies in computer networks.

\section{Acknowledgement}

The authors wish to thank Bart Rouwenhorst for his contribution to the results of the case study.

\section{References}

[1] King, R.E. and Wilson, C., 1991. A review of automated guided vehicle systems design and scheduling. Prod. Planning Control, 2: 44-51.

[2] Co, C.G. and Tanchoco, J.M.A., 1991. A review of research on AGVS vehicle management. Eng. Costs Prod. Economics., 21: 35-42.

[3] Srinivasan, M.M., Bozer, Y.A. and Cho, M., 1994. Tripbased material handling systems: throughput capacity analysis. IIE Trans., 26: 70-89.

[4] Egbelu, P.J. and Tanchoco, J.M.A., 1986. Potentials for bidirectional guide-path for automated guided vehicle based systems. Int. J. Prod. Res., 24: 1075-1097.

[5] Gaskins, R.J. and Tanchoco, J.M.A., 1987. Flow path design for automated guided vehicle systems. Int. J. Prod. Res., 25: 667-676.

[6] Maxwell, W.L. and Muckstadt, J.A., 1982. Design of automated guided vehicle systems. IEE Trans., 14: 114-124.

[7] Goetz, W.G. and Egbelu, P.J., 1990. Guide path design and location of load pickup/drop-off points for an automated guided vehicle system. Int. J. Prod. Res., 28: 927-941.

[8] Bozer, Y.A. and Srinivasan, M.M., 1991. Tandem configurations for automated guided vehicle systems and the analysis of single vehicle loops. IIE Trans., 23: 72-82.

[9] Sinriech, D. and Tanchoco, J.M.A., 1992. Impact of empty vehicle flow on performance of single-loop AGV systems. Int. J. Prod. Res., 30: 2237-2252.

[10] Tanchoco, J.M.A. and Co, C.G,, 1991. A controller for AGVs with multiple-load carrying capacities. Proc of the XIth Int. Conf. on Production Research. Taylor \& Francis, London, pp. 125-128.

[11] Ozden, M., 1988. A simulation study of multiple-loadcarrying automated guided vechicles in a flexible manufacturing system. Int. J. Prod. Res., 26: 1353-1366.

[12] Van den Berg, Y., 1993. The operational control of a multiple-load AGV. unpublished note, Group Production and Operations Management, Department of Mechanical Engineering, University of Twente, Enschede, The Netherlands.

[13] Egbelu, P.J. and Tanchoco, J.M.A., 1984. Characterization of automated guided vehicle dispatchning rules. Int. J. Prod. Res., 22: 359-374.

[14] Occeña, L.G. and Yokota, T., 1991. Modeling of an automated guided vehicle system (AGVS) in a just-in-time (JIT) environment. Int. J. Prod. Res., 29: 495-511. 
[15] Egbelu, P.J., 1987. Pull versus push strategy for automated guided vehicle load movement in a batch manufacturing system. J. Manufacturing Systems, 6: 209-221.

[16] Sabuncuoglu, I. and Hommertzheim, D.L., 1992. Dynamic dispatching algoritm for scheduling machines and automated guided vehicles in a flexible manufacturing system. Int. J. Prod. Res., 30: 1059-1079.

[17] Blazewicz, J., Eiselt, H.A., Finke, G., Laporte, G. and Weglarz, J., 1991. Scheduling tasks and vehicles in a flexible manufacturing system. Int. J. Flexible Manufacturing Systems, 4: 5-16.

[18] Jaikumar, R. and Solomon, M.M., 1990. Dynamic scheduling of automated guided vehicles for a certain class of systems. J. Manufacturing Systems, 9: 315-323.

[19] Bodin, L., Golden, B., Assad, A. and Ball, M., 1983. Routing and scheduling of vehicles and crews. Comput. Oper. Res., 10: 63-211.

[20] Stein, D.M., 1978. Scheduling dial-a-ride transportation systems. Transportation Sci., 12: 232-249.
[21] Clarke, G. and Wright, J.W., 1964, Scheduling of vehicles from a central depot to a number of delivery points. Oper. Res., 12: $568-581$.

[22] Huang, J., Palekar, U.S. and Kapoor, S.G., 1993. A labeling algorithm for the navigation of automated guided vehicles. J. Eng. Industry, 115: 315-321.

[23] Adams, J., Balas, E., and Zawack, D., 1988. The Shifting Bottleneck Procedure for job-shop scheduling. Management Sci., 34: 391-401.

[24] Zijm, W.H.M. and Nelissen, E.H.L.M., 1990. Scheduling a flexible machining centre. Eng. Costs Prod. Economics, 19: $249-258$.

[25] Johnson, M.E., and Brandeau, M.L., 1993. An analytic model for design of a multi-vehicle automated guided vehicle system. Management Sci., 39: 1477-1489.

[26] Askin, R.G. and Standridge, C.R., Modeling and Analysis of Manufacturing Systems, Wiley, New York. 